\title{
Multicentric and multifocal versus unifocal breast cancer: is the tumor-node-metastasis classification justified?
}

\author{
Tobias M. Weissenbacher $\cdot$ Madeleine Zschage $\cdot$ Wolfgang Janni $\cdot$ \\ Udo Jeschke - Thomas Dimpfl - Doris Mayr • Brigitte Rack • \\ Christian Schindlbeck $\cdot$ Klaus Friese $\cdot$ Darius Dian
}

Received: 15 April 2009/Accepted: 23 April 2010/Published online: 8 May 2010

(C) Springer Science+Business Media, LLC. 2010

\begin{abstract}
For classification of breast cancer (BC), tumor-node-metastasis (TNM) staging has been considered state of the art for more than 50 years. The T category is well defined, and in multicentric and multifocal tumors, tumor size is assessed by the largest tumor focus. The aim of this study was to compare multicentric/multifocal tumor spread in breast cancer with unifocal disease and to evaluate the diagnostic relevance of multifocality. A retrospective analysis was performed on survival related events in a series of 5,691 breast cancer patients between 1963 and 2007. By matched-pair analysis, patients were entered into two comparable groups of 288 patients after categorizing them as having multifocal/multicentric or unifocal breast cancers. Matching criteria were tumor size, grading, and hormone receptor status, which were equally distributed between both
\end{abstract}

T. M. Weissenbacher - M. Zschage - U. Jeschke · B. Rack ·

C. Schindlbeck · K. Friese · D. Dian

I. Frauenklinik, Klinikum der Ludwig-Maximilians-Universitaet,

Munich, Germany

T. Dimpfl

Klinikum Kassel, GmbH IBZ-Interdisziplinäres

Brustzentrum Mönchebergstr, Kassel, Germany

W. Janni

Frauenklinik, Heinrich-Heine-Universitaet,

Düsseldorf, Germany

D. Mayr

Pathologisches Institut, Ludwig-Maximilians-Universität,

Munich, Germany

T. M. Weissenbacher $(\square)$

Department of Gynecology and Obstetrics, Klinikum Innenstadt, Ludwig-Maximilians-University Munich, Maistr. 11, 80337 Munich, Germany

e-mail: tobias.weissenbacher@med.uni-muenchen.de groups $(P=1.000$ each). Disease free survival and the occurrence of relapse or of metastatic disease were evaluated. Cox's regression analysis was used for multivariate analysis. In the unifocal group, the mean breast cancerspecific survival time was 221.6 months as opposed to 203.3 months in the multicentric/multifocal group $(P<$ $0.001, \log$-rank test). The occurrence of local relapse and distant metastasis was significantly increased in the multifocal group in comparison to the unifocal equivalent group $(P<0.001$ and $P<0.003$, respectively). Cox regression analysis for multivariate analyses demonstrated focality and centricity to be highly significant predictors for reduced overall survival $(P=0.016)$, local relapse $(P=0.001)$ and distant metastasis $(P=0.038)$. Tumor size, histopathological grading, hormone receptor status, and staging of lymph nodes are well-established prognostic parameters. Additionally, the number of foci should be considered as an independent prognostic parameter, which is currently not reflected in the TNM classification. We conclude that multicentric/multifocal $\mathrm{BC}$ is an independent $\mathrm{BC}$ risk factor and should be included in the risk assessment by re-evaluating the current TNM classification of the UICC.

Keywords Breast cancer - Overall survival . Multicentric · Multifocal · Prognostic factor · Prognosis

\section{Introduction}

For breast cancer classification, tumor-node-metastasis (TNM) staging has been the standard for more than 50 years. During this time, however, the classification has changed substantially. In 2003, the 6th Edition of the TNM classification was established [1-4]. The prognostic relevance of the $\mathrm{T}$ category is a constant factor in regard to the 
current and past classification [4]. Survival of patients with breast cancer depends on two different types of prognostic factors: tumor size as a marker of tumor biology and as a time-dependent phenomenon, and biological factors (i.e., histological grade), which represent tumor aggressiveness [5]. Other prognostic factors are the estrogen and progesterone receptor status as well as the number of mitotic figures per ten high power fields (MF/10HPF) [6, 7]. The treatment follows worldwide prevailing suggestions, including those of the TNM system. Not only is the TNM classification changing, treatment recommendations and the treatments themselves have been modified as well.

Breast conserving treatment, once a controversial issue, is now an established alternative to modified radical mastectomy for surgically manageable breast cancer. Established prognostic factors [5, 8, 9] as well as new possible factors, such as the E-cadherin transcriptional repressor Snail or the c-Jun activation domain binding protein-1 (Jab1), are multifunctional signaling proteins that are controversially discussed in the literature [10-12]. Despite these ongoing modifications, tumor size in multicentric/multifocal tumors is only assessed by the largest tumor focus and does not reflect the cumulative tumor volume.

The aim of this study was to compare the prognosis of multicentric/multifocal tumors with unifocal tumors with apparently identical tumor size according to TNM staging.

\section{Patients and methods}

\section{Patients}

Based on a consecutive patient cohort consisting of 5,691 patients who were documented and surgically treated for primary breast cancer between 1963 and 2007, two equivalent groups of 288 early breast cancer patients with multicentric/multifocal versus unifocal tumors were selected using a matched-paired analysis (see "Statistical analysis" section below). Study sites were the Departments of Gynecology of the University Hospitals in BerlinCharlottenburg (1963-1987) and Munich-Innenstadt (1987-2007).

Work up modalities for verifying unifocality and specifying data on multifocality/centricity included clinical examinations, ultrasonics, and $\mathrm{x}$-ray. In addition, a nuclear magnetic resonance imaging (NMRI), galactography, or pneumocystography were performed if necessary.

Data were contemporaneously gathered for the unifocal and multicentric/multifocal tumors. To be eligible, patients were required to be free of disease, and they must have been treated at the study sites at the time of primary diagnosis of resectable breast cancer. The tumor stage at primary diagnosis was classified according to the UICC
TNM classification [13]. The Bloom-Richardson system was used for histopathological grading, and match criteria were tumor size, grading, and hormone receptor status, all of which were equally distributed in both groups $(P=1.0)$. We consciously disclosed the lymph node statuses (LNS) as matching criteria to evaluate differences in LNS in respect to focality.

\section{Treatment}

The primary surgical treatment consisted of either breast conservation or modified radical mastectomy. Routine axillary dissections were performed on lymph nodes of levels I and II, while those of level III were excised only in cases expressing macroscopic metastatic of the lower levels. For the diagnosis of lymph node metastasis, single embedded lymph nodes were screened at up to three levels. Telecobalt radiation therapy was administered in patients treated with breast conservation. Chest-wall irradiation was given to all mastectomy patients before 1979, while patients after this date received chest-wall irradiation in cases with extensive lymphangiosis carcinomatosa surrounding the tumors. The median absorbed dose in the target volume was 50 Gy fractionated in 25 fractions in all patients who received irradiation.

According to the guidelines of the Cancer Treatment Center of Munich, patients received chemotherapy in cases of lymph node involvement. Depending on the hormone receptor status they received adjuvant endocrine therapy with tamoxifen $(20 \mathrm{mg}-30 \mathrm{mg} /$ day $)$. The guidelines for chemotherapy and cytostatic regime were changing dramatically during the observation time of the study. In our patient series, the most common type of chemotherapy consisted of six cycles of $\mathrm{CMF}$, cyclophosphamide $\left(600 \mathrm{mg} / \mathrm{m}^{2}\right.$ body-surface area), methotrexate $\left(40 \mathrm{mg} / \mathrm{m}^{2}\right)$, and 5-fluoruracil $\left(600 \mathrm{mg} / \mathrm{m}^{2}\right)$ every 21 days. However, this regime was not investigated as adjuvant systemic chemotherapy in a randomized trial. Tamoxifen was given to hormone receptor-positive postmenopausal patients. Premenopausal patients also received GnRH-analogs in the later years of the 44-year time period. Aromatase inhibitors were used in case of contraindications.

Patients were evaluated over a very long period (44 years). As mentioned above, during this time surgical, radiation oncology, and chemotherapy treatment options changed dramatically. However, we did not separate into age-dependent cohorts. Due to the large number of patients, we assumed a normal distribution. A matchedpair-analysis was used to determine the overall and relapsefree survival by comparing multicentric/multifocal versus unifocal breast cancer. Different prognostic factors were correlated with breast cancer-specific survival and relapsefree survival using a multivariate Cox regression analysis. 


\section{Statistical analysis}

Data were entered into the database in a coded fashion. An error-correcting and plausibility check was performed before starting the statistical analysis. Contingency tables were constructed, analyzed by a chi-square test to evaluate differences of possible non-parametric data. Because of the uneven distribution of prognostic factors in our original patient group of 576 cases that met the match criteria, a matched-pair analysis was performed. A total of 288 pairs of patients, each consisting of one patient with unifocal and one with multicentric/multifocal tumor lesions, were selected according to the highest degree of equivalence in the following hierarchical and sequential order: tumor size at the time of primary diagnosis, histopathological grading, and hormone receptor status. Each parameter was required to have a $P$ value $>0.50$ to achieve intergroup homogeneity. We deliberately matched criteria characterizing the patients at the time of primary diagnosis.

Data quality was controlled by verifying all reported immunocytochemical and histopathological results, event reports during follow-up, and by reexamining the original data files. As a starting point the day of the primary operation and as an end point occurrence of relapse or cancer-associated death were chosen. Kaplan-Meier life table curves were constructed to estimate survival and were compared using the log-rank test. Cox's regression analysis was used for multivariate analyses, and $P$ values less than 0.05 were considered significant. The computer software "Statistical Package for the Social Sciences 15.0" (SPSS Inc., Chicago, USA) was used.

\section{Follow-up}

Patients attended the oncology outpatient clinic of our institution at least every 3 months during the first 2 years after primary diagnosis or after a local recurrence, every 6 months for the following 2 years, and every year thereafter. Local recurrence was defined as chest-wall recurrence in case of mastectomy and in-breast recurrence in patients with breast saving treatment. Ipsilateral lymph node metastases were defined as local recurrence.

Follow-up included a clinical examination and yearly mammography. Chest radiography, ultrasound of the liver, bone scan, blood tests, and a computed tomography of the appropriate region were performed according to tumor manifestation and/or symptoms. If patients sought care elsewhere, data were collected from the family physicians or gynecologists in charge. All deaths were verified by referring to the German Cancer Register [14] and classified according to the cancer-associated cause. The mean observation time was 70 months (5.8 years, range: 19-321 months).

\section{Results}

A total of 288 pairs, each consisting of one patient with unifocal disease and one patient with multifocal/-centric breast cancer, were created by matched-pair analysis to achieve statistical balance of the major prognostic factors between both groups. All Match criteria (tumor size, grading, and hormone receptor status) were equally distributed in both groups $(P=1.0)$. Furthermore, no significant difference was found between the two groups in terms of systemic therapy and primary operation. The primary patient characteristics of both groups are shown in Table 1. According to the histological tumor type, there was a significant difference in the incidence of invasive lobular cancer in the multifocal group in comparison to the unifocal group. All the 92 patients had an invasive lobular cancer, 79 of which had a multifocal/-centric disease, whereas only 13 had a unifocal breast cancer. These results were similar concerning invasive ductal tumors. Regarding systemic therapy, 297 patients are included in the multicentric/multifocal group and 295 are in the unifocal group which exceeds the total number of 288. Some patients received only endocrine or only cytostatic therapy, whereas some individuals underwent both types of systemic therapy. Furthermore, patients with multicentric/multifocal disease were demonstrated to have a higher rate of chemotherapy compared to patients with unifocal breast cancer. In all, 101 women $(35.1 \%)$ received cytostatic therapy in the multicentric/multifocal group, whereas only 65 patients $(22.6 \%)$ did so in the unifocal group. In contrast, 61 patients $(21.2 \%)$ with unifocal disease received endocrine therapy as compared to only 51 patients (17.7\%) in the multicentric/multifocal group. Considering these treatment variations, a subgrouping was done for the survival curves (Figs. 4, 5, 6, 7).

\section{Univariate analysis}

At the mean follow-up time of 70 months (5.8 years, range: 19-321 months), the mean breast cancer-specific survival time of 221.6 months in patients with unifocal disease (206.7-236.4, 95\% The confidence Interval [CI]) was significant higher compared to patients with multicentric/multifocal tumors (203.3 months, 184.1-222.5, 95\% CI, $P<0.0001$, log-rank test) (Fig. 1). In patients with a unifocal tumor, the relapse-free survival was 205.9 months $(190.7-221.2,95 \% \mathrm{CI})$, as opposed to 169.6 months $(150.8-188.5,95 \%$ CI, $P<0.0001$, log-rank test) in patients with multicentric/multifocal disease. Twenty-one of 288 patients (7.3\%) with unifocal disease developed a local recurrence compared to 50 of 288 $(17.4 \%)$ with multifocal tumors $(P<0.0001)$. Occurrence of distant metastases was observed in 61 multicentric/ 
Table 1 Patient characteristics at primary diagnosis

\begin{tabular}{|c|c|c|c|}
\hline & Multicentric/multifocal (\%) & Unifocal (\%) & $P$ Value \\
\hline Number of patients & 288 & 288 & \\
\hline Age & 54.0 & 57.4 & .001 \\
\hline Lymph node metastases & & & .0001 \\
\hline Absent (N0) & $139(48.3)$ & $168(58.3)$ & \\
\hline 1-3 axillary LNM (pN1) & $60(20.8)$ & $79(27.4)$ & \\
\hline 4-9 axillary LNM (pN2) & $47(16.3)$ & $26(9.0)$ & \\
\hline 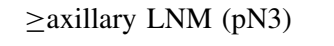 & $42(14.6)$ & $15(5.2)$ & \\
\hline Histological type & & & .0001 \\
\hline Ductal & 207 (71.9) & $274(95.1)$ & \\
\hline Lobular & $79(27.4)$ & $13(4.5)$ & \\
\hline Ductal-lobular & $2(0.7)$ & & \\
\hline Other & & $1(0.3)$ & \\
\hline Menopausal status & & & .001 \\
\hline Premenopausal & $138(47.9)$ & $108(37.5)$ & \\
\hline Postmenopausal & $150(52.1)$ & $180(62.5)$ & \\
\hline Primary operation & & & .047 \\
\hline Breast conserving & $124(43.1)$ & $145(50.3)$ & \\
\hline Mastectomy & $164(56.9)$ & $143(49.7)$ & \\
\hline Radiotherapy & & & .001 \\
\hline Performed & $118(41.0)$ & $167(58.0)$ & \\
\hline Not performed & $170(59.0)$ & $121(42.0)$ & \\
\hline Systemic therapy & & & .027 \\
\hline None & $145(50.3)$ & $169(58.7)$ & \\
\hline Endocrine & $51(17.7)$ & $61(21.2)$ & \\
\hline Cytostatic & $101(35.1)$ & $65(22.6)$ & \\
\hline Local recurrence & & & .001 \\
\hline No recurrence & $238(82.6)$ & $267(92.7)$ & \\
\hline Recurrence & $50(17.4)$ & $21(7.3)$ & \\
\hline Distant metastasis & & & .004 \\
\hline Absent & $227(78.8)$ & $252(87.5)$ & \\
\hline Distant metastasis & $61(21.2)$ & $36(12.5)$ & \\
\hline
\end{tabular}

multifocal diseased patients, as compared to 36 patients in the unifocal group (Figs. 2, 3).

The univariate Kaplan-Meier analyses of patients with multicentric/multifocal diseases receiving chemotherapy $(N=101)$ was significantly different from the unifocal group $(N=65)(P=0.001)$. Regarding hormonal therapy, no statistical correlation was identified $(P=0.34$, Log rank). Fifty-one patients within the multicentric/ multifocal group received hormone therapy, compared to 61 in the unifocal group. Figures 4, 5, 6, and 7 illustrate the differences in overall survival comparing focality according to hormone therapy and chemotherapy (Figs. 4, $5,6,7)$. Overall survival was significantly better in patients with unifocal breast cancer compared to multicentric/multifocal disease, either with or without hormone therapy $(P=0.002)$. Regarding chemotherapy, overall survival was different in patients with multicentric/ multifocal disease compared to unifocal disease but the results did not reach statistical significance $(P=0.07)$ (Figs. 4, 5, 6, 7).

\section{Multivariate analysis}

All of the major established prognostic factors for overall survival as well as for the occurrence of local relapses or metastases (lymph node metastases, grading, tumor size, age, hormone receptor status, chemotherapy, hormonal therapy, and radiation) were included in the multivariate analysis to evaluate the impact of focality. For the Cox multivariate regression analysis regarding breast cancerspecific survival, local or systemic relapse showed that multicentricity/multifocality is a significant independent predictor of reduced breast cancer-specific survival as well as of reduced relapse-free survival (Tables 2, 3). Relapse 


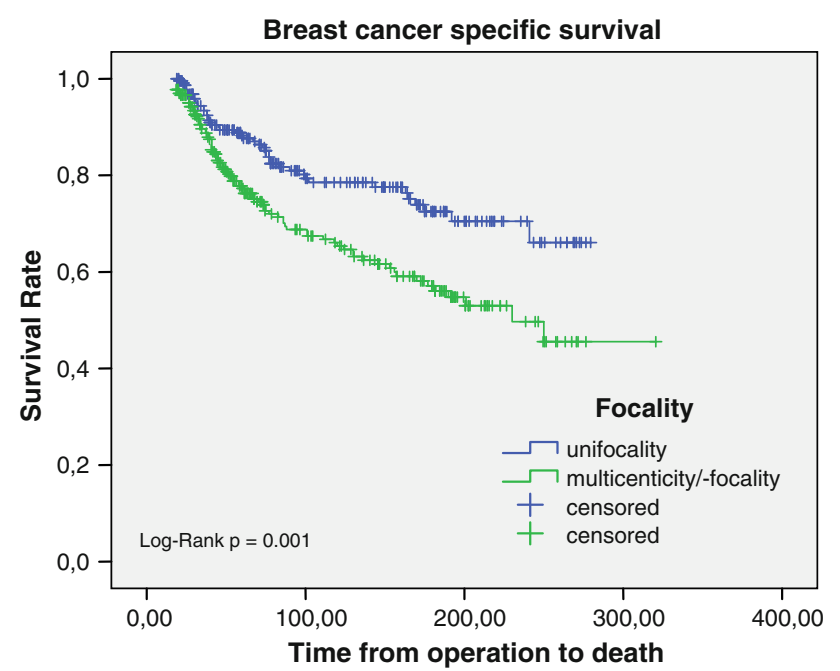

Fig. 1 Kaplan-Meier breast cancer-specific survival analysis among patients with multicentric/Multifocal and unifocal breast cancer in the time of follow-up (in months [mon])

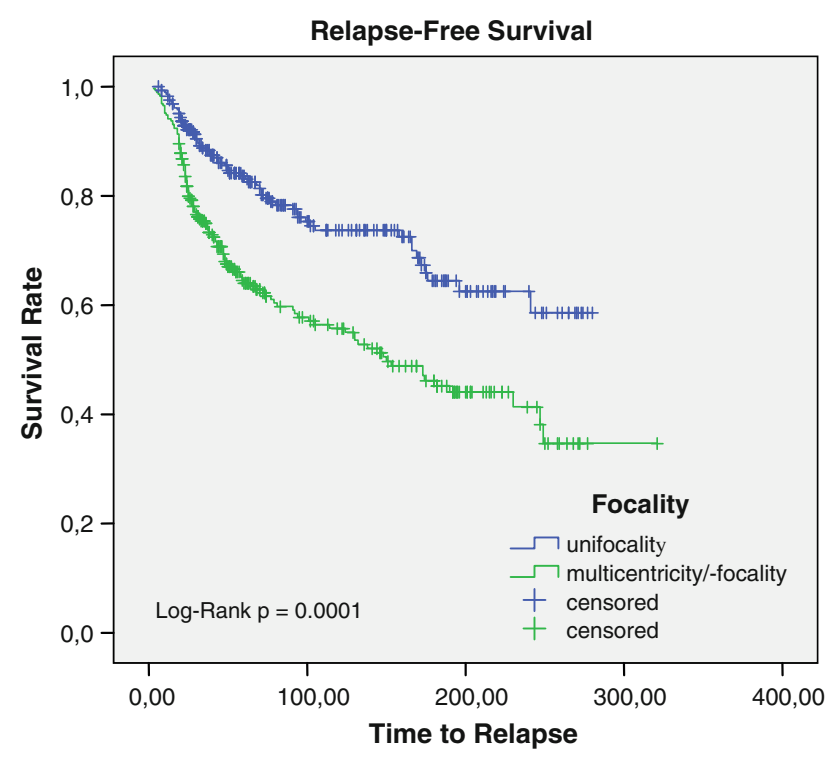

Fig. 2 Kaplan-Meier for relapse-free survival analysis among patients with multicentric/Multifocal and unifocal breast cancer in the time of follow-up (in months [mon])

was defined as overall recurrence, including local, regional, and distant recurrence of disease,

Tumor size, grading, and lymph node status were also significant independent predictors. Hormone receptor status and age failed to demonstrate significance for the prediction of overall survival $(P=0.80$ for progesterone receptor status, $P=0.43$ for estrogen receptor status, and $P=0.21$ for age). In addition, chemotherapy and radiation showed no statistical significance by multivariate analysis in regard to overall survival. Hormonal therapy was significant concerning overall survival $(P=0.002)$. Concerning

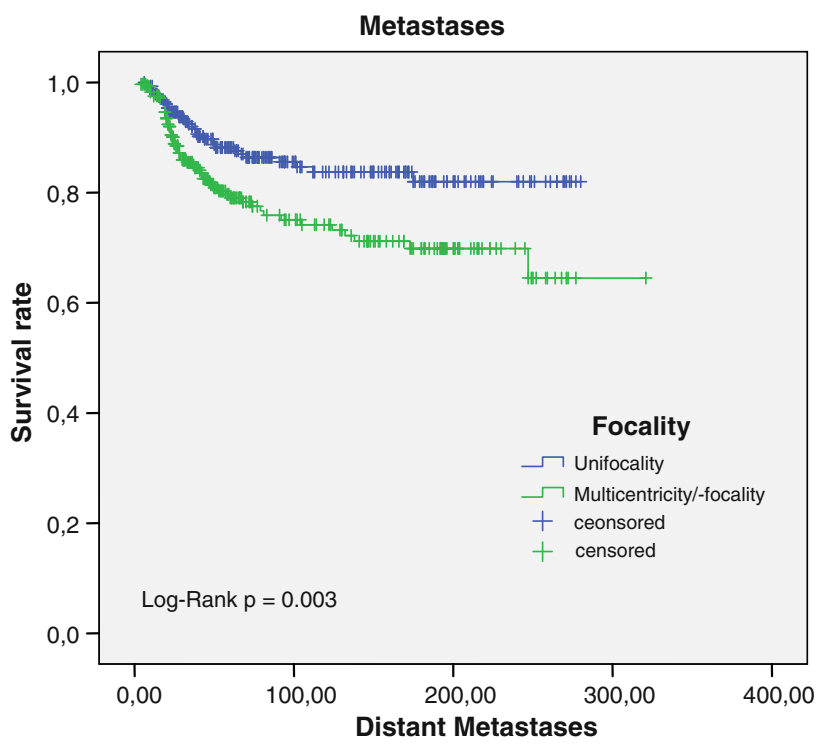

Fig. 3 Kaplan-Meier occurrence of distant metastasis analysis among patients with multicentric/Multifocal and unifocal breast cancer as the time of follow-up (in month [mon])

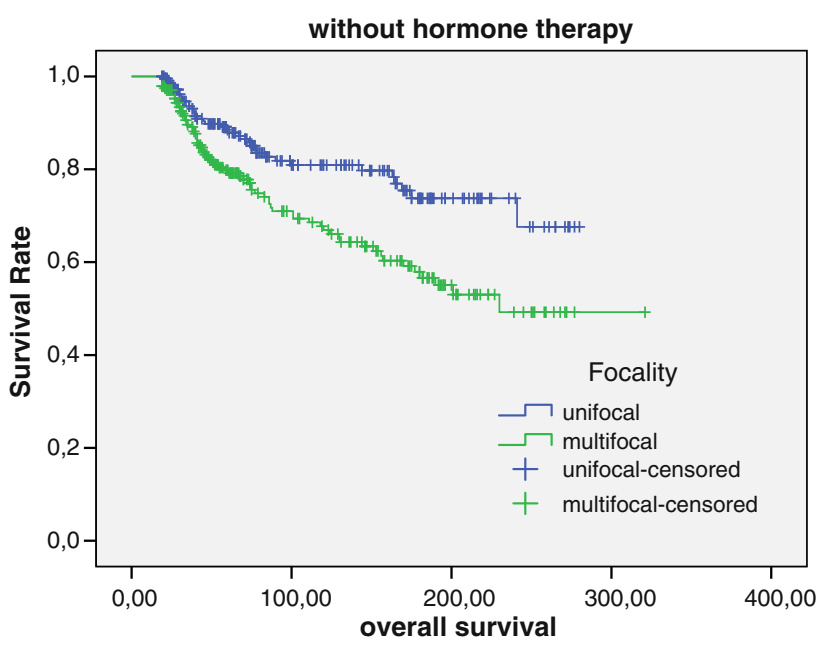

Fig. 4 Kaplan overall survival without hormone therapy analysis among patients with multicentric/multifocal and unifocal breast cancer as the time of follow-up (in month [mon])

disease recurrence, neither chemotherapy, hormonal therapy nor radiation showed any significance (Tables 2,3).

\section{Discussion}

The TNM classification has changed over time to provide more reliable prognostic information. Tumor size, lymph node involvement, and metastases are the key elements taken into account. Especially, LNS is known to be a relevant factor convening disease free survival (DFS) and the 


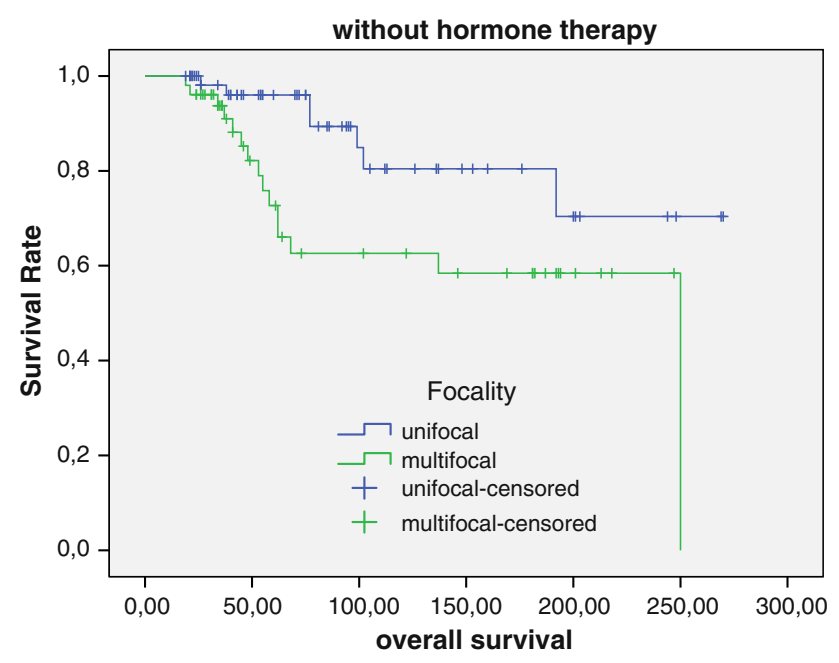

Fig. 5 Kaplan-overall survival with hormone therapy analysis among patients with multicentric/multifocal and unifocal breast cancer as the time of follow-up (in month [mon])

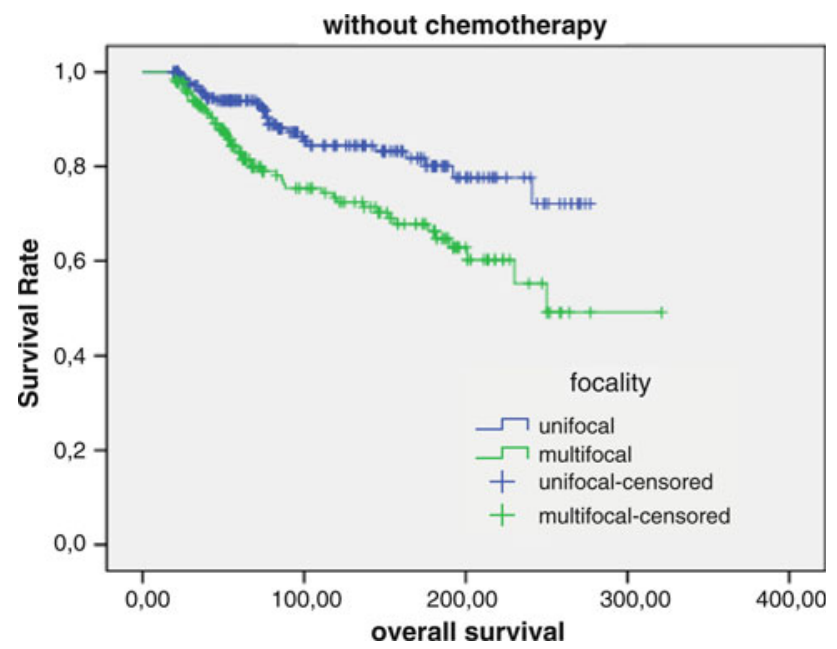

Fig. 6 Kaplan-Meier for overall survival without chemotherapy analysis among patients with multicentric/multifocal and unifocal breast cancer as the time of follow-up (in month [mon])

development of metastases. In our study, we consciously utilized this parameter as matching criteria to evaluate differences in LNS with respect to focality. Looking at the multivariate Cox Regression Analysis in our study, LNS is shown to be a highly significant risk factor for all evaluated events.

Several authors suggest using the TNM 6 classification [4, 8, 15-18], including the definition of micrometastases. A transformation from the current rigid categorization into a more flexible system is also recommended [4] because several additional factors should be considered during the decision-making process.

Diagnosis and treatment have also changed over the years for multicentric/multifocal breast tumors, although at

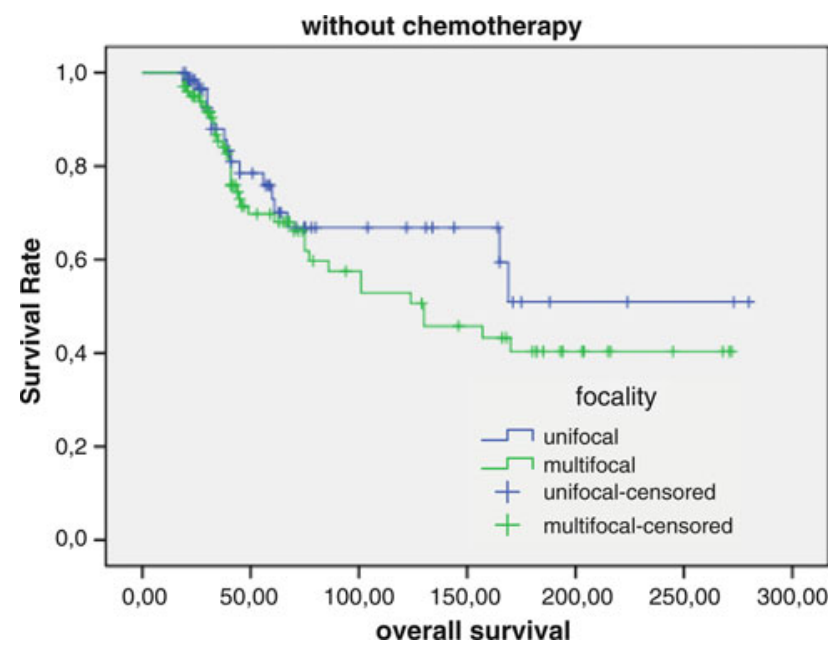

Fig. 7 Kaplan-Meier for overall survival with chemotherapy analysis among patients with multicentric/multifocal and unifocal breast cancer as the time of follow-up (in month [mon])

Table 2 Multivariate Cox regression analysis for breast cancer-specific survival

\begin{tabular}{llll}
\hline Variables & Significance & Wald statistics & HR \\
\hline Tumor size $^{\mathrm{a}}$ & .013 & 6.2 & 1.02 \\
Lymph node metastases $^{\mathrm{a}}$ & .0001 & 36.8 & 1.08 \\
Grading $^{\text {Focality }}$ & .044 & 4.04 & 2.42 \\
Chemotherapy & .016 & 5.8 & 1.57 \\
Hormonal therapy & 0.07 & & 0. \\
Radiation & 0.002 & & 0.002 \\
Age & 0.157 & & \\
\end{tabular}

a At time of primary treatment

$H R$ Hazard ratio

Table 3 Multivariate Cox regression analysis for overall recurrence

\begin{tabular}{llll}
\hline Variables & Significance & Wald statistics & HR \\
\hline Tumor size $^{\mathrm{a}}$ & .005 & 7.8 & 1.08 \\
Lymph node metastases $^{\mathrm{a}}$ & .0001 & 37.8 & 1.07 \\
Radiation therapy $_{\text {Focality }}$ & .028 & 4.8 & .71 \\
Chemotherapy & .0001 & 12.1 & 1.74 \\
Hormonal therapy & 0.761 & & \\
Radiation & 0.107 & & \\
Age & 0.151 & & \\
\hline
\end{tabular}

a At time of primary treatment

$H R$ Hazard ratio

a much slower pace. Breast conserving treatment is now an established alternative to radical mastectomy. As for tumors with more than one lesion, treatment suggestions are currently changing. Many researchers support the 
extension of conservative surgery for multicentric/multifocal tumors [19-22]. Nevertheless, the indications should be accurately applied as some authors report a higher rate of recurrence after conservative treatment [23].

In terms of TNM classification, however, the tumor size in multicentric/multifocal breast carcinomas still refers to the diameter of the largest lesions. This study examined two groups of patients with multicentric/multifocal and unifocal breast cancer. Both groups were well balanced for the established prognostic indicators-tumor size, grading, and hormone receptor status at the time of presentationand equally distributed in both groups $(P=1.0)$. Our longterm findings, with a median follow-up of 70 months (5.8 years), demonstrated that multicentricity/multifocality are significant independent predictors for reduced breast cancer-specific survival as well as reduced relapse-free survival.

These findings are consistent with several studies [24-28]. To our knowledge, however, no other study to date has examined the overall survival as well as the relapse-free survival. Other studies have shown, that if aggregate diameters are used, unifocal and multifocal breast carcinomas are similar with respect to the frequency of regional lymph node metastasis. The currently used algorithms, which employs the diameter of the largest nodule, result in the downplaying of multifocal breast carcinomas due to the underestimation of actual tumor size $[24,25]$. It has been suggested that studies should indicate the number of macroscopically recognizable tumors in parentheses after the T classification [26, 27]. Andea et al. [25] showed that, when a combined diameter assessment was used, the frequency of lymph node positivity was not significantly different in multifocal versus unifocal cases. Coombs and Boyages [29] confirmed this finding. Concerning this investigation, multicentric and multifocal breast cancer is associated with increased nodal involvement compared to similar unifocal disease and the tendency of breast tumors to metastasize is a reflection of the total tumor load [29]. When looking only at the largest diameter, an understaging of multifocal breast carcinomas results [25]. Evaluating patients characteristics (Table 1), $30.9 \%$ of $\mathrm{BC}$ patients in the multicentric/mulitfocal group were classified as $\mathrm{pN} 2$ and $\mathrm{pN} 3$ in our study. This was more than twice as much as in the unifocal group with $14.2 \%$. This might result in facilitating treatment decisions and, subsequently, in the outcome of the multicentric/mulitfocal group. All of the previously mentioned studies reveal a clear coherence between tumor load and lymph node involvement in a subsequently poor outcome.

In contrast, Fajdic et al. [30] did not show an association between size and increased risk for a poor prognosis of multicentric carcinomas. This study included 296 breast cancer patients, but only 29 were histopathologically proven as multicentric tumors, and significantly lower survival rates were shown in all advanced tumor stages. The finding of this study is reliable for lower tumor stages. By retrospectively analyzing 706 patients who received neoadjuvant anthracycline-based chemotherapy, clinically detecting multicentric/multifocal disease in this study also failed to predict an inferior outcome [31]. Even when cancer stages were reaching from levels I-III, the prognostic value was masked by the neoadjuvant chemotherapy, which does not collectively reflect the findings of our study. The results of this study should, therefore, be interpreted with caution.

Vlastots et al. [28] examined 284 patients and detected multicentric breast cancer in 60 patients. There was a significant difference between groups in estrogen receptor and axillary lymph node positivity, but these did not significantly contribute to the outcome of the multivariate analysis. Therefore, multicentricity in this study did not increase the risk of poor outcome. In contrast, our study showed no significant differences regarding hormone receptor status and patient age for the prediction of overall survival. We found a reduced breast cancer-specific survival as well as reduced relapse-free survival (Figs. 1,2). These findings are in contrast to Vlastots et al. [28].

The tendency of breast tumors to metastasize [29] is a reflection of the total tumor load. Failure to measure the additional tumor burden provided by multiple small foci may underestimate the disease. This may deny patients the opportunity of adjuvant therapies if the contribution of the smaller foci to the incidence of node positivity and survival is ignored. This hypothesis is supported by our findings. The factors correlated to multicentricity were shown to be, in a retrospective study by Anastassiades et al. [32] the age of the patient, the size, and the histological type of the tumor, levels of the progesterone receptors more than 50 $\mathrm{fmol} / \mathrm{mg}$ of protein, and the amount of solid tissue in the breasts. In contrast, our results are not comparable due to the fact that the above elements represent our match criteria.

Many studies point out an increased risk for metastatic dissemination and, therefore, poorer outcome, a finding that is not currently reflected in the TNM classification [23-27]. As mentioned above, the prognostic relevance of the $\mathrm{T}$ category is a constant factor in regard to current and past classification [4] and survival of patients with breast cancer depends on several prognostic factors. Tumor size as a marker of tumor biology and as a time-dependent phenomenon, represents a very important prognostic factor, but might, according to our results, be misrepresented. On the basis of the presented data, we conclude that in breast cancer multicentric/multifocal disease is associated with a worse prognosis compared to unifocal disease with an identical tumor size. In summary, the combined tumor 
volumes appear to be more decisive rather than only the largest tumor diameter. The total tumor volume seems to be of decisive importance rather than the largest tumor diameter. We hypothesize that multicentric/multifocal BC is an independent $\mathrm{BC}$ risk factor and should be included in the risk assessment by re-evaluating the current TNM classification of the UICC.

Acknowledgment We would like to thank Dr. Steven S. Witkin (Weill Cornell Medical College, New York, USA) for his help with the manuscript.

\section{References}

1. Benson JR, Weaver DL, Mittra I, Hayashi M (2003) The TNM staging system and breast cancer. Lancet Oncol 4(1):56-60

2. Cserni G, Kulka J (2003) New TNM classification of breast tumors. Orv Hetil 144(32):1563-1568

3. Escobar PF, Patrick RJ, Rybicki LA, Weng DE, Crowe JP (2007) The 2003 revised TNM staging system for breast cancer: results of stage re-classification on survival and future comparisons among stage groups. Ann Surg Oncol 14(1):143-147

4. Veronesi U, Viale G, Rotmensz N, Goldhirsch A, Rethinking TNM (2006) breast cancer TNM classification for treatment decision-making and research. Breast 15(1):3-8

5. Bundred NJ (2001) Prognostic and predictive factors in breast cancer. Cancer Treat Rev 27(3):137-142

6. Dabakuyo TS, Bonnetain F, Roignot P, Poillot ML, Chaplain G, Altwegg T, Hedelin G, Arveux P (2008) Population-based study of breast cancer survival in Cote d'Or (France): prognostic factors and relative survival. Ann Oncol 19(2):276-283

7. Younes M, Lane M, Miller CC, Laucirica R (1997) Stratified multivariate analysis of prognostic markers in breast cancer a: preliminary report. Anticancer Res 17(2B):1383-1390

8. Duraker N, Caynak ZC (2005) Prognostic value of the 2002 TNM classification for breast carcinoma with regard to the number of metastatic axillary lymph nodes. Cancer 104(4):700-707

9. Garcia-Vilanova Comas A, Garcia Vilanova A, Fuster-Diana E, Martinez-Alzamora N, Fernandez-Tena J, Garcia-Vilanova Comas J, Garcia-Vilanova Comas M (2006) Prognostic value of the interpectoral lymph nodes in breast cancer. A 20 -year survival study. Clin Transl Oncol 8(2):108-118

10. Wanami LS, Chen HY, Peiro S, Garcia de Herreros A, Bachelder RE (2008) Vascular endothelial growth factor-A stimulates snail expression in breast tumor cells: Implications for tumor progression. Exp Cell Res 314(13):2448-2453

11. Wang J, Barnes RO, West NR, Olson M, Chu JE, Watson PH (2008) Jab1 is a target of EGFR signaling in ER-alpha negative breast cancer. Breast Cancer Res 10(3):R51

12. Yildirim Y, Gunel N, Coskun U, Sancak B, Bukan N, Aslan S, Cetin A (2008) Serum big endothelin-1 levels in female patients with breast cancer. Int Immunopharmacol 8(8):1119-1123

13. Sobin LH, Hermanek P, Hutter RV (1988) TNM classification of malignant tumors. A comparison between the new (1987) and the old editions. Cancer 61(11):2310-2314

14. Altmann U, Haeberlin V, Tafazzoli A, Dudeck J (1997) Application of a standard methodology for the development of messages and aspects of realization in the area of tumour documentation. Stud Health Technol Inf 43 Pt B:776-780

15. Gusterson BA (2003) The new TNM classification and micrometastases. Breast 12(6):387-390

16. Johnson A (2006) TNM classification Veronesi et al. Breast 15(5):580

17. Savran VR, Fetsych TH, Savran VV, Tril OV, Myshakivs'kyi OM (2006) Stages of breast cancer. Lik Sprava 3:11-17

18. Singletary SE, Greene FL (2003) Revision of breast cancer staging: the 6th edition of the TNM classification. Semin Surg Oncol 21(1):53-59

19. Gentilini O, Botteri E, Rotmensz N, Da Lima L, Caliskan M, Garcia-Etienne CA, Sosnovskikh I, Intra M, Mazzarol G, Musmeci S et al. (2009) Conservative surgery in patients with multifocal/multicentric breast cancer. Breast Cancer Res Treat 113(3):577-583

20. Gentilini O, Veronesi P, Gatti G, Intra M, Mazzarol G, Trifiro G, Bassi F, Caliskan M, Da Lima L, Galimberti V et al (2007) Conservative approach for breast cancer. The experience of the European Institute of Oncology. Minerva Chir 62(6):447-458

21. Spinelli C, Berti P, Ricci E, Miccoli P (1992) Multicentric breast tumour: an anatomical-clinical study of 100 cases. Eur J Surg Oncol 18(1):23-26

22. Wakabayashi T, Tsuchiya SI, Asano G (1995) Unilateral multicentric breast carcinoma studied by whole mammary gland serial sectioning. Breast Cancer 2(2):91-98

23. Fowble B, Yeh IT, Schultz DJ, Solin LJ, Rosato EF, Jardines L, Hoffman J, Eisenberg B, Weiss MC, Hanks G (1993) The role of mastectomy in patients with stage I-II breast cancer presenting with gross multifocal or multicentric disease or diffuse microclassifications. Int J Radiat Oncol Biol Phys 27(3):567-573

24. Andea AA, Bouwman D, Wallis T, Visscher DW (2004) Correlation of tumor volume and surface area with lymph node status in patients with multifocal/multicentric breast carcinoma. Cancer 100(1):20-27

25. Andea AA, Wallis T, Newman LA, Bouwman D, Dey J, Visscher DW (2002) Pathologic analysis of tumor size and lymph node status in multifocal/multicentric breast carcinoma. Cancer 94(5): 1383-1390

26. Sobin LH, Greene FL (2003) Pathologic analysis of tumor size and lymph node status in multifocal/multicentric breast carcinoma. Cancer 97(5):1364 (author reply 1365)

27. Sobin LH, Greene FL (2004) Multifocal/multicentric breast carcinoma. Cancer 100(11):2488-2489 (author reply 2489)

28. Vlastos G, Rubio IT, Mirza NQ, Newman LA, Aurora R, Alderfer J, Buzdar AU, Singletary SE (2000) Impact of multicentricity on clinical outcome in patients with T1-2, N0-1, M0 breast cancer. Ann Surg Oncol 7(8):581-587

29. Coombs NJ, Boyages J (2005) Multifocal and multicentric breast cancer: does each focus matter? J Clin Oncol 23(30):7497-7502

30. Fajdic J, Bukovic D, Gugic D, Habek M, Hojsak I, Bukovic N, Herman I, Stanceric T (2003) Unilateral multicentric breast cancer. Coll Antropol 27(2):693-698

31. Oh JL, Dryden MJ, Woodward WA, Yu TK, Tereffe W, Strom EA, Perkins GH, Middleton L, Hunt KK, Giordano SH et al (2006) Locoregional control of clinically diagnosed multifocal or multicentric breast cancer after neoadjuvant chemotherapy and locoregional therapy. J Clin Oncol 24(31):4971-4975

32. Anastassiades O, Iakovou E, Stavridou N, Gogas J, Karameris A (1993) Multicentricity in breast cancer. A study of 366 cases. Am J Clin Pathol 99(3):238-243 